\section{Anaesthesia for the patient with cardiac disease}

John H. Tinker MD

\section{Basic coronary physiology}

Before we can discuss the effects of anaesthesia on the coronary circulation, it seems pertinent to discuss the basic control mechanisms involved in the coronary circulation. The coronary circulation is one of if not the most "responsive" circulations in the body. The final determinant of flow to any given region of myocardium is the metabolic demand of that region. Unfortunately, although the metabolic demand of a given region might pose a certain requirement, numerous factors may prevent that metabolic demand from being satisfied. These factors include coronary artery disease, excessive heart rate, rapidly changing afterload, dysrhythmias, as well as various external "tonal" influences such as sympathetic activity and hormonal influences from the adrenal glands and elsewhere.

It was commonly taught that coronary oxygen extraction was nearly complete on a given passage through the myocardium, i.e., the maximal amount of available oxygen would be extracted. Coronary mixed-venous oxygen saturation was considered to be usually if not always as low as practically possible, namely less than 50 per cent, with a $\mathrm{PO}_{2}$ in the low 20's. However, resting coronary venous oxygen saturation is not usually that low and therefore the "first line of defense" against a sudden increase in oxygen demand of the myocardium is simply for an increase in extraction of oxygen from the blood already passing through it.

Next, coronary vasodilation occurs. The final determinant of coronary vasodilation in a particular cardiac region is local demand. This local demand produces adenosine, which is one of the agents important in regional dilatation, together with other factors such as "endothelial relaxant factor," etc. Also, external influences play an extremely important role. The carotid sinus reflex is a tachycardic stimulus in response to a lowered carotid arterial pressure and this reflex produces a large decrease in coronary vascular resistance when arterial pressure decreases. This is important in the interpretation of the studies mentioned below, because it is difficult to maintain constant arterial pressure. The question arises as to whether peripheral vascular effects, namely lowered arterial pressure, or intrinsic pharmacologic properties of the anaesthetic itself, contribute to the coronary vasodilation observed. Suffice it to say that these reflexes affecting the heart rate are the most important external determinants of overall coronary blood flow and exert second by second control over coronary vascular resistance, at least in a global fashion.

Local factors, reflexes, and neural influences as well as endocrine influences all play roles in the regulation of the coronary circulation.

\section{Coronary steal}

"Cononary steal" is a catchy term with several shades of meaning. If there is a collateral-dependent zone of myocardium, blood must flow to that zone through another zone which is more directly connected to a coronary artery. It is conceiyable that a direct pharmacologic vasodilator might decrease the normal tone of the "primary" area of myocardium thus "stealing" blood away from the collateral-dependent zone.

Another "model" of coronary steal is slightly different. One myocardial zone is supplied by an artery which has multiple stenoses, such that the vascular tone in that zone is minimal, but no ischaemia is present, while the other zone is supplied by an artery which has considerably fewer stenoses and so vascular tone is present in that zone. If both of the small arteries supplying these zones are branches of a larger vessel, direct pharmacologic vasodilation will result in "stealing blood away from" the zone which cannot dilate despite the absence of functioning collaterals.

Coronary stealing has been demonstrated with nitroprusside and with adenosine. It is probably impossible to demonstrate coronary steal in vivo in man because of the invasive nature of the methods that must be used to determine regional coronary blood flow. In the absence of determinations of coronary blood flow, it is not possible to be certain that a given occurrence of myocardial ischaemia was necessarily due to the preccding development of coronary steal. After all, hypotension combined with tachycardia, reflex or otherwise, can reduce myocardial blood supply in a particular critical region to the level of

Department of Anesthesia, The University of Iowa Hospitals and Clinics, Iowa City, Iowa, 52242. 
the production of myocardial ischaemia, without invoking any such exotic mechanism as coronary steal. Also it is becoming increasingly obvious that some if not much of the development of ischacmia in the presence of critical coronary stenoses is probably due to the formation of small thrombi. In the future, we may look back on our frantic attempts to control "demand" with a somewhat jaundiced eye. It is possible that future therapeutic and preventive efforts will be focused on the problem of microvascular thrombi.

\section{Anaesthesia and coronary steal}

In 1983, Reiz et al.,' in a study involving 21 unpremedicated patients scheduled for coronary bypass surgery who had severe coronary artery disease of varying types, speculated that isoflurane anaesthesia might have been associated with the production of signs of ischaemia in ten of the 21 patients, by a mechanism involving pharmacologic indiscriminate coronary vasodilation, i.e., steal. In this study, unpremedicated patients, with a group average mean arterial pressure on arrival in the operating room of $110 \mathrm{mmHg}$ were then given an anaesthetic which reduced the group average mean arterial pressure to $68 \mathrm{mmHg}$, over a relatively short time. The authors contended that this led to ischaemia due to coronary steal, although the drop in mean arterial pressure, and the reflex tachycardia induced thereby would have been a massive stimulus for coronary vasodilation. Despite these other possibilities, the authors contended that the mechanism of the resultant ischaemia was coronary steal. This led to the conclusion that isoflurane was a direct coronary vasodilator. Other explanations include the possibility that such a precipitous drop in perfusion pressure might be inhibited from reflex coronary vasodilation by the presence of other anaesthetics. Indeed, halothane and enflurane are more effective in blocking carotid sinus baroreflexes than is isoflurane. In summary, the problem is to separate the direct pharmacologic actions of an anaesthetic from its peripheral and/or reflex effects. If the coronary vasodilation is due to peripheral dilation-induced hypotension, and/or baroreflex-induced coronary vasodilation, then one would not expect such indiscriminate vasodilation as to cause clinically relevant steal.

\section{Four "models" for the study of coronary steal vs anaesthetics}

\section{The human model}

The Reiz et al. ${ }^{\prime}$ paper stimulated other human studies, including those of Moffitt et $\mathrm{al}^{2}$ und Tarnow et al. ${ }^{3}$ In the Moffitt $e t$ al. studies, coronary sinus flow and oxygen contents were measured. Again it was observed that isoflurane anaesthesia appeared to produce coronary dilatation in excess of that produced by relatively equiva- lent concentrations of halothane and enflurane. The studies of both Reiz et al. ${ }^{1}$ and Moffitt et al. ${ }^{2}$ with halothane and enflurane tend to show that as these two agents reduce oxygen demand, both of the whole body and the myocardium and that coronary blood flow decreases appropriately. In contrast, this does not seem to be the case with isoflurane. Although most of these studies have not found increased coronary blood flow during situations of less oxygen demand created by anacsthesia with isoflurane, they have found greater preservation of control coronary blood flows seen during the awake state, indicating that isoflurane is somewhat of a direct coronary vasodilator. The dispute lies in the area of whether or not such apparent preservation of coronary blood flow, i.e., a greater decrease in coronary vascular resistance with isoflurane for a given set of oxygen demand conditions compared with halothane or enflurane, constitutes a direct coronary vasodilatory stimulus sufficient to produce clinically relevant coronary steal.

A study by Tarnow et al., ${ }^{3}$ also in humans, illustrates the difficulty of this dilemma. Patients with coronary artery disease who had angina pectoris were electrically paced to tachycardia-induced angina, while awake. The heart rate level at which each patient developed angina was recorded, as was the electrocardiogram. Next, the patients were anaesthetized with isoflurane and then paced to tachycardia again. During pacing-induced tachycardia under isoflurane anaesthesia, ECG changes similar to those which occurred during awake angina did not appcar until higher levels of tachycardia were induced. Obviously, the patients could not complain of angina but they seemed to tolerate greater levels of tachycardia during isoflurane anaesthesia. Tarnow et al ${ }^{3}$ concluded, in sharp contrast to the studies of Reiz et al.' and Moffitt et al., ${ }^{2}$ that isoflurane protected patients with coronary artery disease against the development of ischaemia.

There are numerous difficulties with human studies. First, coronary blood flow must be measured with a reverse thermodilution coronary sinus catheter, which has some potential problems. First, it is pushed into the coronary sinus an arbitrary distance by the operator. The further one pushes it into the sinus, the more it occludes the flow in that vessel. There are no valves in the coronary venous system, so that if flow in the coronary sinus is impeded, the flow will be diverted elsewhere, usually to the right ventricular thebesian venous channels, and the diverted flow will not be measured with the coronary sinus catheter. Another problem is that, in some patients, the great coronary vein does not empty into the coronary sinus but rather empties directly separately in the right atrium and thus its flow will not be measured. Under the best of circumstances, the coronary sinus only contains about 70-75 per cent of the total coronary blood flow. Another problem with this method of measuring is that the 
atrium gets in the way. When the atrium beats, it not only pushes blood down into the right ventricle, but also backward into the coronary sinus. This means that the reverse thermodilution mechanism of the coronary sinus catheter will have atrial blood washed over it at intervals corresponding to heart rate. If heart rate at the beginning of the study period is not the same as it is during the experimental part, $i, e_{1}$, the anaesthetic, then the accuracy of the coronary sinus blood flow measurements will be in question. This is important, because it is based on these apparently better preserved coronary blood flows that Reiz et al. ' and Moffitt et al. ${ }^{2}$ contend that isoflurane is a direct coronary vasodilator, although these patients did have some degree of reflex tachycardia.

I do not believe that the weight of evidence from the human studies has thus far been convincing that isoflurane, in clinical concentrations, can lead to myocardial ischaemia via a mechanism of coronary steal. There is no doubt in my mind that the internists' adage "keep the blood pressure up, don't let the heart rate go up, keep the oxygen levels up," is true and I am certain that we all try to do this. I think it is well understood by anaesthetists that if you fail in the above objective, in a patient with reduced coronary vascular reserve, you run the risk of severe ischaemia and its consequences but it is not necessary to invoke a mechanism of coronary steal.

\section{The dog model of Buffington et ai..$^{4,5}$}

Dogs have a naturally collateralized coronary circulation, which is different from the human. This has elicited much criticism of the use of dogs for experiments in involving coronary physiology, but it does permit studies of coronary steal. In other animals which have more humanlike coronary circulations, such as the pig, inducing the animal to produce disease-related coronary collaterals is difficult. In the dog, Buffington's group produced a gradual (approximately one week) occlusion of a major coronary artery using an ameroid (a casein-like substance inside a rigid metal ring) constrictor. This gradual constriction either produces an infaret in the distal area, in which case the animal is excluded from further study, or it produces a collateral-dependent zone distal to the occlusion. This collateral-dependent zone does not have perfect oxygen delivery and therefore not perfect mechanical reserve, but it is alive and functioning. Flow to this collateral-dependent zone originates in another major coronary artery, flowing through that artery's normal area and then, via collaterals, into the collateral-dependent zone.

Buffington's experiments were conducted as follows. Using a cannula in the non-occluded coronary artery, flow in that vessel was held constant at a value determined previously by pilot studies to be consonant with maintenance of normal resting function during "basal" anaesthesia with chloralose. During this "basal" anaesthetic, control regional blood flows were measured by injecting microspheres into the cannulated coronary artery. This allowed determination of relative flow in the normal area and the collateral area and the determination of a collateral to normal flow ratio. Next, at this same overall flow. either halothane or isoflurane was added to the "basal" chloralose anaesthetic in concentrations of $1 \mathrm{MAC}$. When steady-state anaesthesia was achieved, a different type of microsphere was again injected to determinc the collatcral to normal flow ratios again. It is important to point out that this experiment was done during constant flow, produced via an external pump. During the basal anaesthesia conditions, the collateral to normal flow ratios were approximately 0.7 . During $1 \mathrm{MAC}$ halothane anaesthesia, this ratio was not significantly reduced. During 1 MAC isoflurane anaesthesia, the collateral to normal flow ratio decreased to about 0.4 . Because overall flow was constant, this is incontrovertible eviderce that steal had occurred.

Unfortunately, the perfusion pressures at which these two ex periments (i.e., halothane vs isoflurane) were done were not the same at all. Control perfusion pressures were approximately $55 \mathrm{mmHg}$ and were only reduced to 51 $\mathrm{mmHg}$ with halothane, but were reduced to $41 \mathrm{mmHg}$ with isoflurane. The authors chose "equiMAC" concentrations, $1 \mathrm{MAC}$ each of halothane and isoflurane, rather than producing a "dose-response" curve. Obviously, if pressures had been held constant between the two anaesthetics, then flow could not have been. The fact that the authors chose to hold flow constant and let pressures vary with the anaesthetics may have influenced the relative outcome of the experiment. It is possible that at equal perfusion pressures there would be little difference between the collateral to normal flow ratios between halothane and isoflurane, though this experiment has not yet been done (it is underway in my laboratory).

These experiments represent the only demonstration of coronary steal with any anaesthetic. Unfortunately they show that at relatively low perfusion pressures, isoflurane is capable of inducing steal. They do not show whether halothane would or would not induce steal at these same low perfusion pressures. Obviously, $\mathrm{I}$ am in disagreement with the use of "equiMAC" concentrations of anaesthetics when studying the coronary circulation. If equiMAC concentrations represent equal cerebral depressive doses, this is not the way anaesthetics are clinically administered, because they are used to control haemodynamics. Thus agents should be studied at equal levels of haemodynamic depression, in this case coronary perfusion pressure before any agent is condemned.

\section{In vitro experiments}

These are the apparently simplest experiments of all. All you need to do is take a section of coronary artery, mount 
it in a bath and measure the tension in that slice after it is constricted with potassium. Next, the suspected coronary vasodilator is added and the effect of that drug is measured. Actually, these experiments are anything but simple, and the work of Bollen et al., ${ }^{5}$ and Blaise et al. ${ }^{7}$ is elegant and complex. This is a model which is widely used for testing drugs for their ability to either constrict or dilate coronary and/or other arteries. When clinically relevant concentrations, in dose-response fashion, of halothane vs isoflurane are tested in this model, using either dog coronary arteries (Blaise et al. $)^{7}$ or pig coronary arteries (Bollen et al ), ${ }^{6}$ surprising results are obtained. Neither halothane or isoflurane are really vasodilators of these coronary arteries. If anything, halothane is a slightly greater vasodilator than is isoflurane.

Criticism of these studies comes from the fact that these use relatively large vessels ( $1-2 \mathrm{~mm}$ diameter). These are epicardial vessels and therefore the fact that halothane and isoflurane are not direct pharmacologic vasodilators of these vessels does not mean that some other small vessels are not dilated by isoflurane (or halothane). One may conclude that if direct pharmacologic vasodilation occurs with isoflurane, it does so at the resistance (arteriolar) level. Nonetheless, these studies do not lend support to the idea that isoflurane might cause coronary steal via direct pharmacologic coronary dilation.

Studies of reactive hyperaemia ${ }^{8-10}$

After temporary (10-15 seconds) but complete occlusion of a coronary artery, its sudden release is followed by a remarkable degree of reactive hyperaemia. The coronary circulation is immensely reactive and the height of the reactive hyperaemia has been contended by Marcus et al. ${ }^{\prime \prime}$ and others to be indicative of the state or reserve of the coronary circulation distal to the point at which occlusion was produced.

Using this concept, we conceptualized that if isoffurane (or halothane) was a direct phanmacologic vasodilator then as increased concentrations of the agent were delivered, the height of the reactive hyperaemia after a ten-second coronary occlusion would be diminished because the agent would have already caused a direct phannacologic coronary vasodilation. ${ }^{8-10}$ Our contention was, therefore, that if either of these agents was associated with relative preservation of the ability of the coronary circulation to dilate in response to a temporary total occlusion, then that agent could not have been very much of a direct pharmacologic vasodilator. It is important to note that a $10-15$ second coronaty occlusion does not damage the myocardium if done repeatedly in careful fashion. Also, this 10-15 second coronary acclusion is the most powerful coronary dilating stimulus known. In short, if there was "anything left to dilate" this stimulus will dilate it.
To our surprise, when we increnentally increased the steady-state end-tidal anaesthetic concentrations, 0.5 MAC at a time, in pigs, measuring the coronary vascular reserve at each of these incremental steady-state concentrations of either balothane or isoflurane, we found that isofturane preserved coronary vascular reserve at least twice as well as did halothane. We do not see how isofluranc could therefore have been acting as a direct coronary vasodilator.

These studies can be criticized as follows. First, we were measuring coronary flow velocity, not coronary flow. It is conceivable that dilation could have increased coronary flow while decreasing the measured velocity. We controlled for this and this study bas now passed extensive peer review so we do not believe that criticism particularly valid. Our experimental model was the previously normal pig, not with diseased coronary arteries. Nonetheless we conclude from this study that we could find no evidence whatsoever that isoflurane was an indiscriminate coronary pharmacologic vasodilator. We also believe that the reactivc hyperaemic method we used is an excellent way to cancel out the peripheral and reflex effects of these anaesthetics, again because a 10-15 second total coronary occlusion is the most powerful coronary dilation stimulus known.

\section{Summary and conclusions}

I have tried to summarize experimental work in human and animal models. This work is complex and difficult to interpret. Clinicians should not take isolated experimental studies and jump to conclusions about clinical use of anaesthetics. Experimenters should not arrive at sweeping conclusions based on a particular experimental study performed under some or other carefully or not so carefully controlled conditions. Editorialists, such as Becker ${ }^{12}$ in a recent issue of Anesthesiology, should not conclude that an anaesthetic is "dangerous," based on these kinds of studies. It is especially invalid to use the term "dangerous" unless the author is willing to present us with a viable, experimentally proven, safer alternative. To tell us that isoflurane is "dangerous" implies that we should switch to some unspecified other agent.

What conclusions can be arrived at based on thesc studies? (1) It is possible to produce coronary steal, in dogs, at constant coronary flow, with isoflurane at 41 $\mathrm{mmHg}$ perfusion pressure. The comparison between halothane and isoflurane is suspect because is was performed at two markedly different coronary perfusion pressures. (2) Myocardial ischaemia can be induced in humans with coronary artery disease when hypotension and tachycardia is permitted during isoflurane anaesthesia. During these conditions, relative preservation of coronary blood flow greater than that which would be expected because of measured or calculated demand has 
been interpreted as meaning that the mechanism was that of coronary steal. I am not convinced that this mechanism needs to be invoked here, but we can conclude from these studies that if hypotension and tachycardia are allowed to occur in patients with coronary artery disease, ischaemia is possible. (3) In isolated in vitro studies, direct pharmacologic vasodilation of the coronary vessels cannot be demonstrated in either dogs or pigs with either isoflurane or halothane. (4) Studies of coronary reactive hyperaemia, i.e., "coronary reserve" show considerably better preservation of said reserve with isoflurane conpared with halothane. These studies were done in previously normal pigs.

Summarizing, it seems to me that it is still valid to do what we have always tried to do in the operating room in patients with coronary artery disease, namely preserve myocardial oxygen supply and not let demand get too far out of hand. It is increasingly clear to me that supply is more important than demand in these patients. This is why I work hard to keep subendocardial perfusion pressure as normal as possible by preventing or treating acute increases in pulmonary capillary wedge pressure. I do this while trying to preserve reasonable levels of mean arterial pressure. It is also important to keep haemoglobin content in mind.

Finally, let me speculate about the future. A wellknown study by Slogoff and Keats ${ }^{13}$ contended that occurrence of myocardial ischaemia in the operating room, in patients with coronary artery disease, was associated with an increased incidence of perioperative myocardial infarction. As Reves has pointed out, their data can be interpreted almost exactly oppositely, namely that a perioperative myocardial infarction will result only about six per cent of the time, despite the fact that ischaemia occurred in the operating room. One should ask why did the infaret occur in those six per cent and not in the other 94 per cent of patients? Increasingly, the answer may be thrombosis. We must become much more sophisticated about understanding, measuring and treating the coagulation states of our patients perioperatively. We must understand what the stress response does to the blood's coagulability in each patient and develop methods of treating or preventing it. We went through a whole decade of intense worries about myocardial oxygen demand. Countless lectures were given showing the famous scale between myocardial oxygen supply demand balance. Since the bottom line is really not ischaemia but infarction, I think our focus must now turn toward what it is that produces the actual infarction. By this I do not mean that we should ignore ischaemia, obviously it must be treated, and I think we know how to treat it now very well. We also are getting better and better at preventing it from occurring in the operating room, but I am less sure that we are getting any better at preventing perioperative myocar- dial infarction. Therefore, I will close with a prediction for the future, namely watch for new developments in the area of studies of the coagulation state of patients in the postoperative period. In the meantime, I remain unconvinced that "coronary steal" induced by any anesthetic, isoflurane or otherwise, will be an important part of our future clinical considerations.

\section{References}

1 Reiz S, Balfors $E$, Sorensen $M$ et al. Isoflurane - a powerful coronary vasodilator in patients with coronary artery disease. Anesthesiology 1983; 59: 91-7.

2 Moffitt E, Barker RA, Glenn JI et al. Myocardial metabolisn and hemodynamic responses with isoflurane anesthesia for coronary arterial surgery. Anesth Analg 1986; 65: 53-61.

3 Tarnow J, Markschies-Hornung A, Schulte-Sasse $U$. Isoflurane improves the tolcrance to pacing-induced myocardial ischemia. Anesthesiology 1986; 64: 147-56.

4 Buffington $C$, Levine A. Effects of isoflurane, halothane and adenosine on regional myocardial flow and function in a canine model of chronic coronary occlusion. Anesthesiology 1986; 65: A6.

5 Buffington C, Romson JL, Levine A et al. Isofluranc induces coronary steal in a canine model of chronic coronary occlusion. Anesthesiology 1987; 66: 280-92

6 Bollen B. Tinker JH, Hermsmeyer K. Halothane relaxes previously constricted isolated porcine coronary artery segments more than isoflurane. Anesthesiology 1987; 66: $748-52$.

7 Blaise $G$, Sill $J$, Nugent $M$ et al. Isoflurane attenuates epicardial coronary artery response to phenylephrine and $\mathrm{PGF}_{2}$ in dogs. Anesth Analg 1986; 65: $\mathrm{S} 20$.

8 Gilbert $M$, Roberts $S$, Biomberg $R$, Tinker J. Greater coronary reserve in swine anesthetized with isoflurane vs halothane. Anesthesiology 1985; 63: A15.

9 Roberts $S L$, Gilbert $M$, Tinker $I H$. Isoflurane has a greater margin of safety than halothane in swine with and without major surgery or critical coronary stenoses. Anesth Analg 1987; 66: 485-91.

10 Cilbert $M$. Greater coronary reserve in swine anesthetized with isoflurane vs halothane. Anesthesiology (in press).

11 Marcus $M$, Wright $C$, Doty $D$ et al. Measurements of coronary velocity and reactive hyperemia in the coronary circulation of hunnans. Circ Res 1981; 49: 877-91.

12 Becker $L$. Is isoflurane dangerous for the patient with coronary artery disease? Anesthesiology $1987 ; 66: 259-61$.

13 Singoff $S$, Keats $A$. Does perioperative myocardial ischemin lead to postopcrative myocardial infarction? Anesthesiology 1985; 62: 107 